\title{
Immuno-oncology: developing integrated approaches toward clinical success of biologics and small-molecule modulators
}

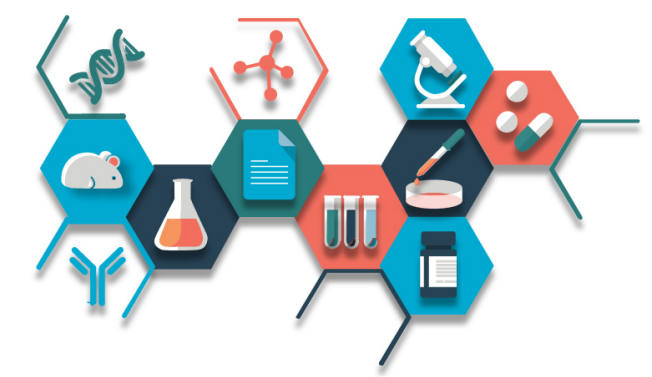

\author{
Shilina Roman*,1, Sanne Holt ${ }^{2} \&$ Julia Schueler ${ }^{3}$ \\ ${ }^{1}$ Associate Director, Biology, Discovery Services Charles River Laboratories, Harlow, UK \\ ${ }^{2}$ Group Leader, Biology, Discovery Services Charles River Laboratories, Leiden, The Netherlands \\ ${ }^{3}$ Research Director, Biology, Discovery Services Charles River Laboratories, Freiburg, Germany \\ *Author for correspondence: shilina.roman@crl.com
}

Immuno-oncology (IO) therapy is an exciting emerging pillar of cancer treatment that embraces the concept of modulating the immune system to recognize tumor cells and target them for destruction by either harnessing the effects of the immune system or preventing the evasion of tumor cells from therapeutic targeting. However, our immune system is constantly in a delicate balance between under-performing immune cells failing to manage pathogens, infections or cancer and over-performing immune cells potentially causing autoimmune disorders or cytokine release storms. Over the last 30 years, 10 has progressed considerably with approvals for the use of various IO therapeutics including vaccines, cytokines, tumordirected monoclonal antibodies, immune checkpoint inhibitors as well as chimeric antigen receptor (CAR) and T-cell receptor (TCR) engineered T-cell therapies.

First draft submitted: 15 November 2019; Accepted for publication: 22 November 2019; Published online: 9 April 2020

\section{Immune checkpoint inhibition}

Immune checkpoint inhibition targets proteins such as the CTLA-4 receptor and the PD-1 receptor along with its ligand PD-L1. CTLA-4 was the first immune checkpoint receptor to be identified and validated as a drug target, and it has progressed into clinical trials for a variety of cancers. Utilizing a combination of checkpoint inhibitors can improve long-term survival rates, increasing them to levels approaching $60 \%$ in some cancer types. The hope is that novel and appropriately scheduled immune checkpoint inhibitor combinations, along with patient stratification strategies, could increase survival rates in the future, bringing them closer to $80 \%$ or pushing them even higher [1].

\section{Cancer vaccines}

Cancer vaccines are designed to stimulate the body's immune system to mount a more robust and effective attack against cancer cells that are already established or circulating in the body with relevant antigens on surfaces of cancer cells, ultimately increasing the specificity of the immune response. In 2010, the US FDA (MD, USA) approved the first cancer vaccine for prostate cancer, Provenge (sipuleucel-T) [2].

Recent advances in cancer vaccines have been made in target selection, vaccine technology and methods for reversing the immunosuppressive mechanisms exploited by cancers, as reviewed by Hollingsworth and Jansen [3]. Studies testing different tumor antigens have revealed target properties that yield high tumor versus normal cell specificity and adequate immunogenicity to affect clinical efficacy.

\section{Adoptive T-cell therapy}

CAR- and TCR-engineered T cells are part of a big wave of immunotherapies showing great promise in cancer clinical trials. Clinical trials using CAR-T cells engineered to recognize B-cell cancers (via a specificity for the B-cell antigen CD19) have shown high rates of response (70-90\%) along response times of unprecedented durability in acute and chronic leukemia [4], with the first two CD19 CAR-T therapies (Kymriah and Yescarta) now approved by the FDA and EMA (Amsterdam, The Netherlands). Solid tumors form an addition challenge due to the lack 


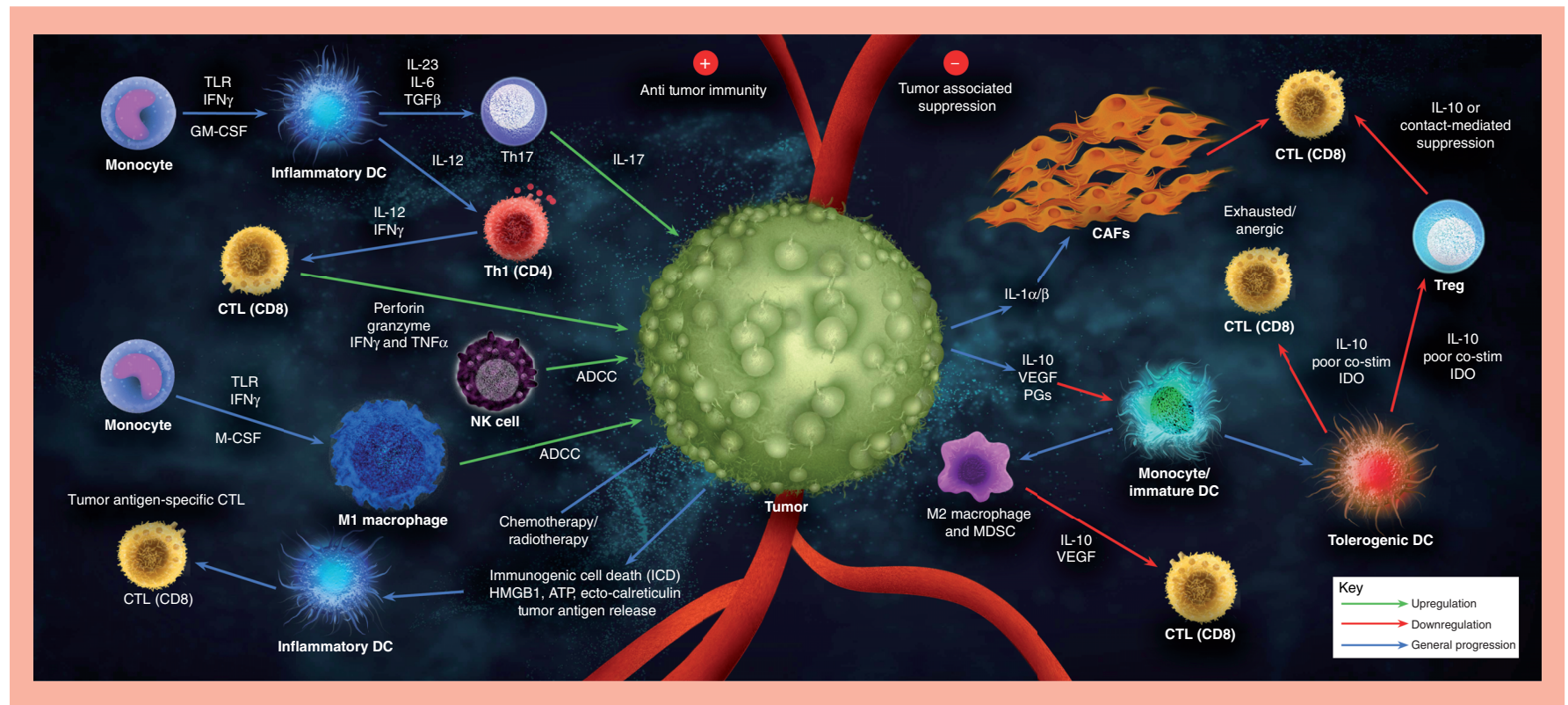

Figure 1. Cell-based assays at Charles River Laboratories.

ADCC: Antibody dependent cell-mediated cytotoxicity; CTL: Cytotoxic T lymphocyte; DC: Dendritic cell; IDO: Indoleamine 2,3-dioxygenase; MDSC: Myeloid-derived suppressor cell; NK: Natural killer cell; TLR: Toll-like receptor.

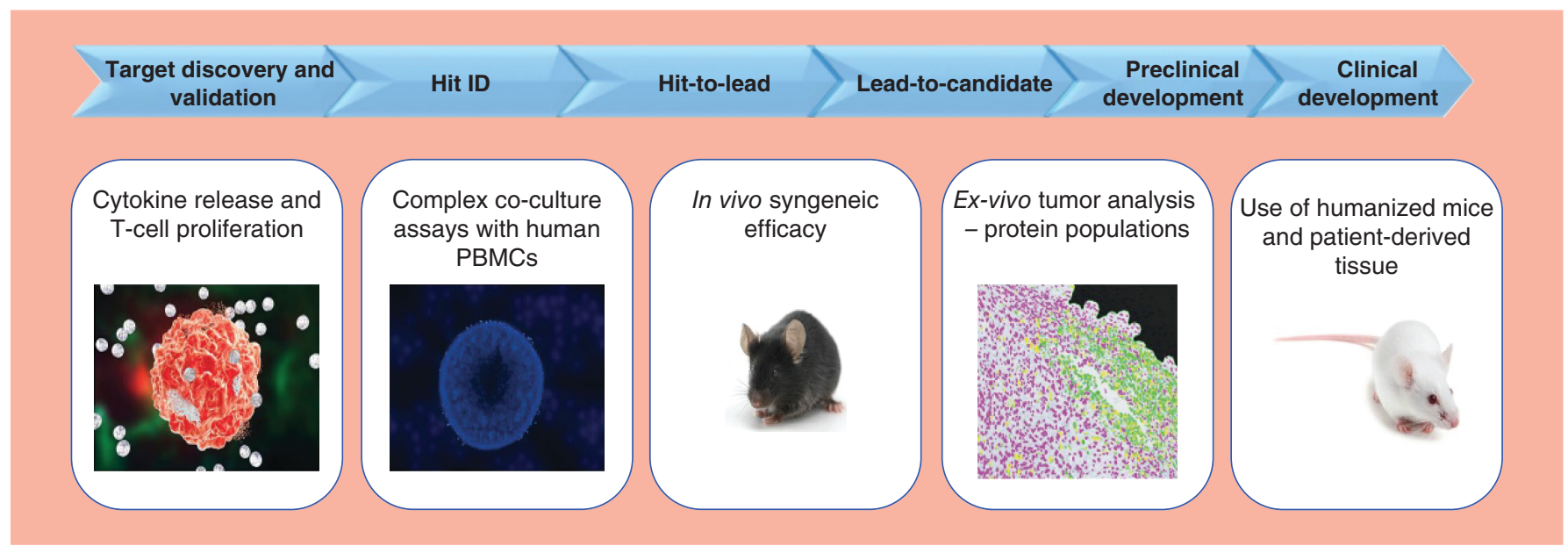

Figure 2. Workflow enabled by Charles River Laboratories' translationally focused immuno-oncology platform.

PBMC: Peripheral blood mononuclear cell.

of tumor-specific target antigens, posing significant safety risks, in other words, on-target on-tumor, on-target off-tumor and off-target toxicities [5]. Currently, solid tumors remain a significant challenge but with ongoing research into the area it is hoped that CAR-T therapies may offer benefits in the future.

On-target off-tumor and off-target effects within cell-based IO therapies, either TCR or CAR-T cells, are an important part in both early-stage development and late-stage testing of the therapeutic product. In silico analysis of target antigen expression can provide some insight into the safety risks of novel T-cell therapies; however, potential T-cell activation and target antigen binding will need to be established in vitro or in vivo.

\section{Immuno-oncology drug discovery platforms at Charles River Laboratories}

Developing IO therapeutics is a challenging area given the complexities of the tumor microenvironment (TME). The TME is a complex network, consisting of the tumor, blood vessels, stromal and immune cells and soluble 


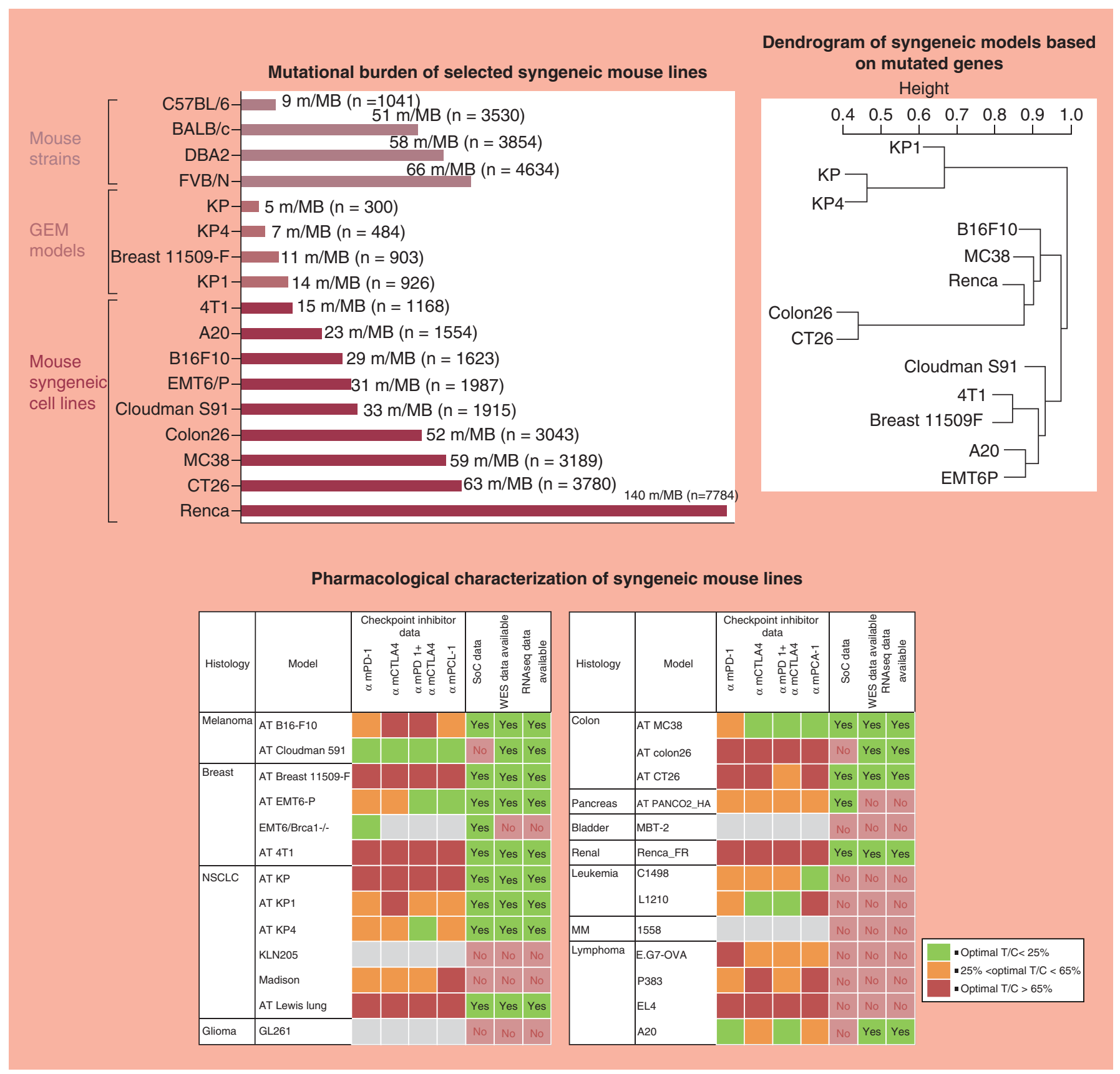

Figure 3. Molecular and pharmacological characterization of syngeneic mouse models. GEM: Genetically engineered mouse NSCL: Non-small-cell lung; T/C: Test/control.

factors [6]. It is now well known that the immune system plays a significant role in combating tumor growth, and many studies have associated raised immune infiltrate with a beneficial outcome in the suppression of cancer growth [7]. Hence, it is critical to test novel immunomodulators in assays involving multiple cell types to assess interactions, to understand the mechanism of action (MOA) and identify biomarkers before moving into the clinic.

At Charles River Laboratories (CRL; MA, USA), we exploit the complex TME observing immunoreactivity in a multitude of cell-based assays to predict MOA, pharmacodynamics and efficacy downstream. We have developed single cell and coculture cell-based assays in 2D and 3D formats to monitor an immunotherapeutic's interaction with specific cell types, such as T cells including CAR-T cells, natural killer cells, macrophages, dendritic cells, neutrophils and fibroblasts. The assays currently available can be found on the CRL website [8]. 
CRL has developed in vitro assays utilizing primary human cells from healthy tissue and/or differentiated induced pluripotent stem cells (iPSC)-derived cells to assess cytotoxicity for engineered TCR and CAR-T therapies. The presence of T-cell mediated cytotoxicity was measured in assays with a panel of target cell types, including the central nervous system, liver, heart, kidneys, lung, skin, vasculature and bone marrow, in coculture with engineered T cells. The primary tissues were selected based on their potential safety risk by either expressing low levels of the target antigen or being major organs to de-risk for off-target effects.

Another much debated topic for T-cell therapies is the balance between affinity, efficacy and safety of the therapy; a CAR-T with lower affinity and efficacy in target antigen binding could still manage tumor burden very efficiently but offer a better safety profile. In vitro assays utilizing cancer cell lines exhibiting various levels of target expression (e.g., low, medium and high), offer a robust and rapid way to establish efficacy and aid lead optimization.

Comprehensive characterization of novel IO compounds using a multitude of such in vitro assays can provide insights into understanding the detailed MOA and identify potential toxicity issues to improve translation to in vivo and ultimately the clinic. To deliver detailed data packages for IO drug discovery projects, the scientists at CRL have established a range of cell-based assays (Figure 1) and a powerful translational IO platform (Figure 2) for progressing biologics or small-molecule modulators of the immune response from in vitro to in vivo assays. CRL also offers technologies to help with the design and development of both CAR-T and bispecific antibodies through our partnership with Distributed Bio [9].

With regard of the inevitable basic role of the immune system in tumor biology, the necessity to use immunecompromised mice might be the most obvious drawback of in vivo patient derived xenograft (PDX) models. Tumor cells are broadly thought to be antigenic due to their multiple mutations in coding exons resulting in a phenotypic make-up reasonably different from the normal cells they evolved [11]. However, most tumors eventually progress and evade the immune surveillance by inducing immune tolerance. The ability to influence the immune system is often related to prognosis: the cytotoxic to regulatory T-cell ratio is a strong prognostic marker in many solid

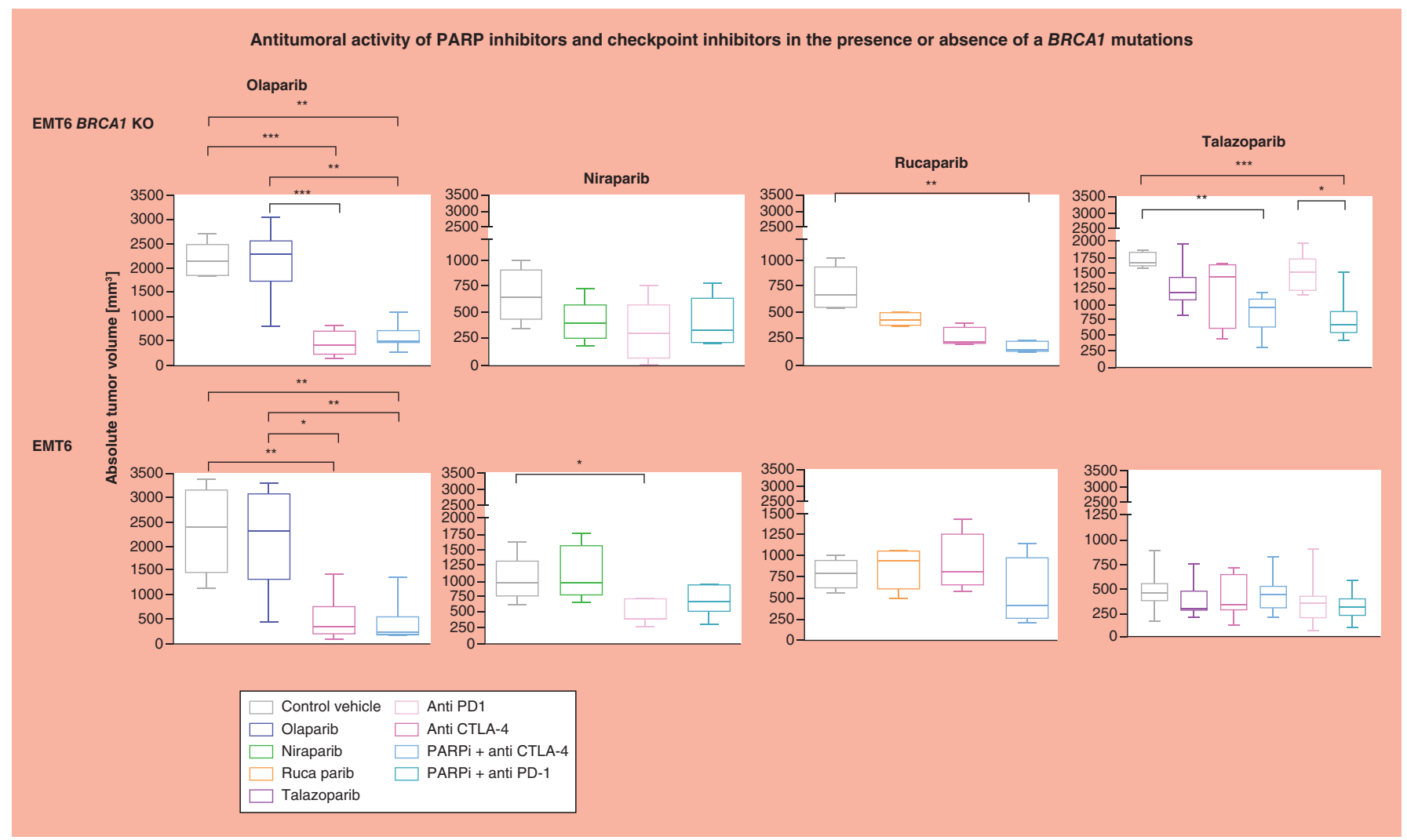

Figure 4. Influence of the BRCA1 mutation on sensitivity toward immune-oncology combination treatments. ${ }^{*} \mathrm{p}<0.05 ;{ }^{* *} \mathrm{p}<0.005 ; * * * \mathrm{p}<0.0005$.

KO: Knock-out; NK: Natural killer; MDSC: Myeloid derived suppressor cells; TIL: Tumor infiltrating lymphocytes. 


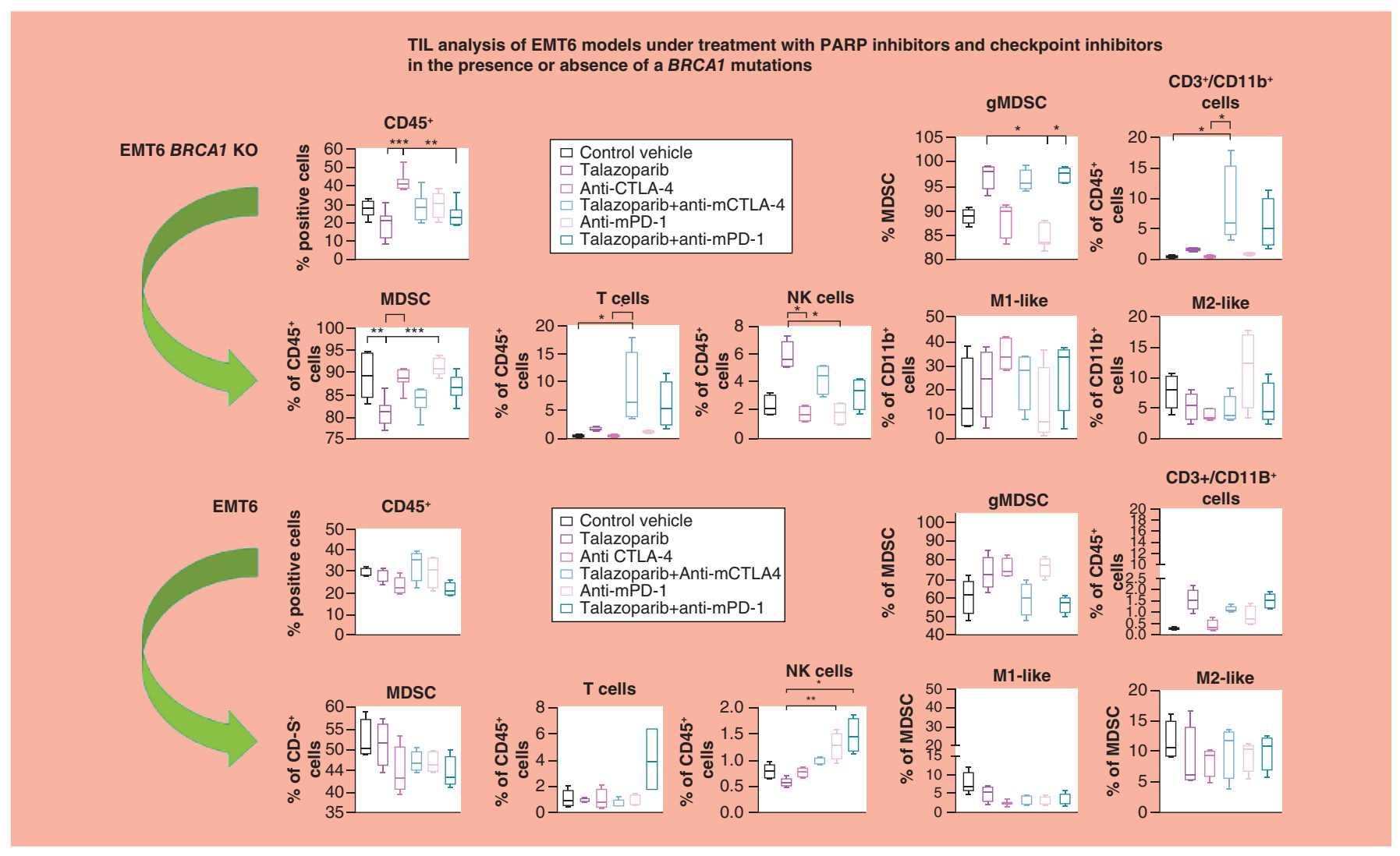

Figure 4. Influence of the (cont.). BRCA1 mutation on sensitivity toward immune-oncology combination treatments. ${ }^{*} \mathrm{p}<0.05 ;{ }^{* *} \mathrm{p}<0.005 ; * * * \mathrm{p}<0.0005$.

KO: Knock-out; NK: Natural killer; MDSC: Myeloid derived suppressor cells; TIL: Tumor infiltrating lymphocytes.

tumor [12]. The fact that macrophage and $\mathrm{CD}_{4}^{+} \mathrm{T}$-cell recruitment following intensive chemotherapy, for example, in breast cancer patients is associated with significantly reduced recurrence-free survival, follows the same line [13].

For the investigation of new innovative modalities, in vivo immune competent as well as immune-compromised mouse models are used at CRL. Both platforms come with a comprehensive preclinical data package. Grafting of neoplastic cell lines established from experimental mice into genetically identical (syngeneic) counterparts enables the examination of tumor-immune interactions in immunocompetent animals. CRL has a panel of $>25$ different syngeneic lines with complementary molecular and pharmacological datasets which allow the development of new compounds and combinations in a time and cost sensitive framework (Figure 3) [14].

Using CRISPR technology, those lines can be modified to closer mimic specific tumor subtypes. The availability of the wild-type as well as the modified lines enables a direct comparison, shedding more light into the tumor biology behind those driver mutations (Figure 4) [15].

The human counterpart of the syngeneic models is the so called 'humanized mice'. They should meet at least three criteria: all lineages and subsets of human hematopoietic cells should develop in the mouse host, in proportions and localizations similar to those observed in healthy humans; these cells should be functional, in vivo; they should allow for testing of therapeutic interventions and faithfully predict the outcome in clinical setting [16]. Two basic approaches are available at CRL: either engraftment is performed with hematopoietic stem cells from different sources or mice are injected with human peripheral blood mononuclear cells. The cotransplantation of human hematopoietic stem cells together with cancer cells seems to enable the coexistence of MHC-mismatched cells without inducing rejection [17], whereas the injection of peripheral blood mononuclear cells into immunecompromised mice is accompanied by the onset of xenograft-versus-host disease [10]. For both humanized in vivo IO platforms, validation datasets including sensitivity data toward checkpoint inhibitors are available (Figure 5) $[18,19]$. 


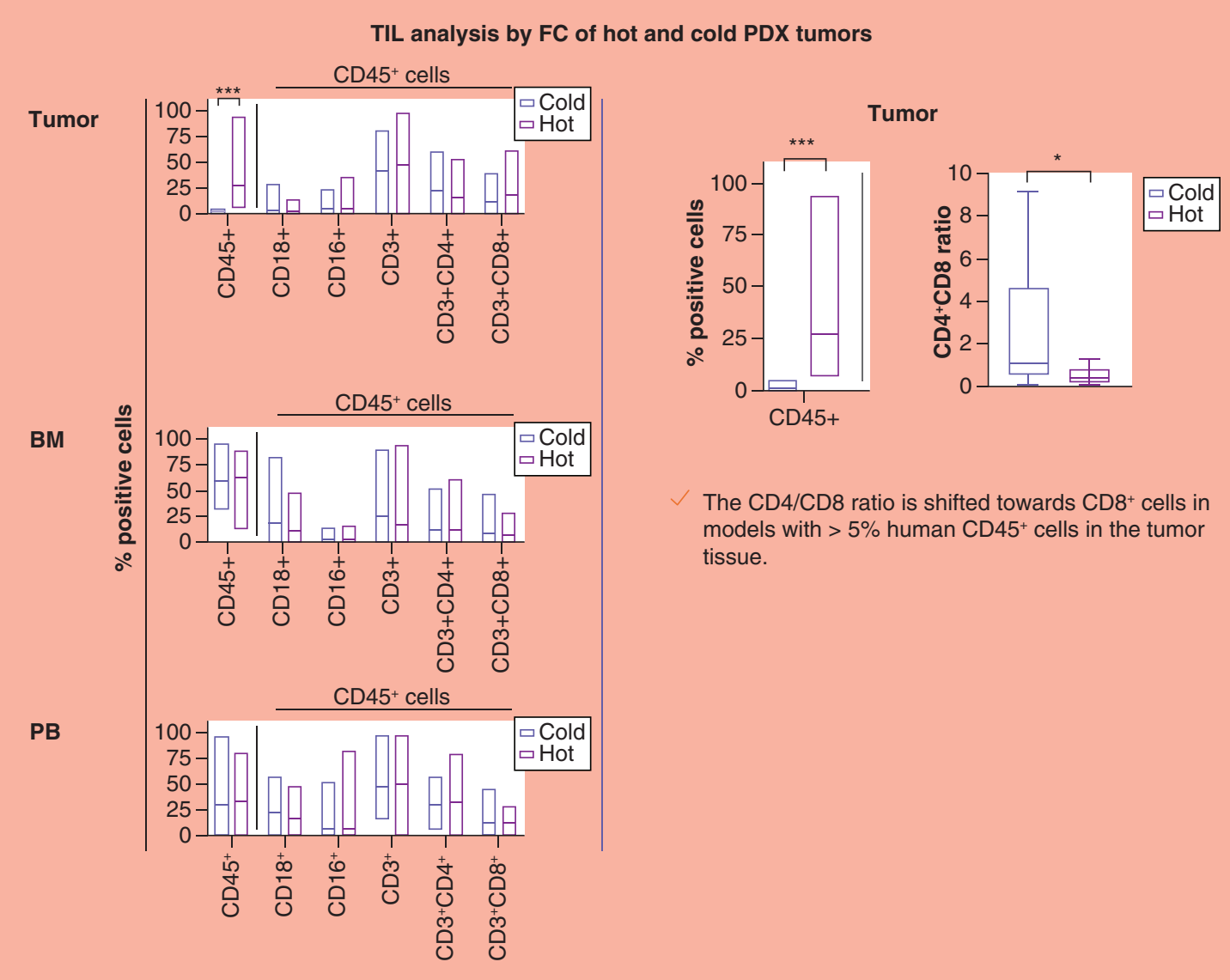

TIL analysis by IHC of hot and cold PDX tumors
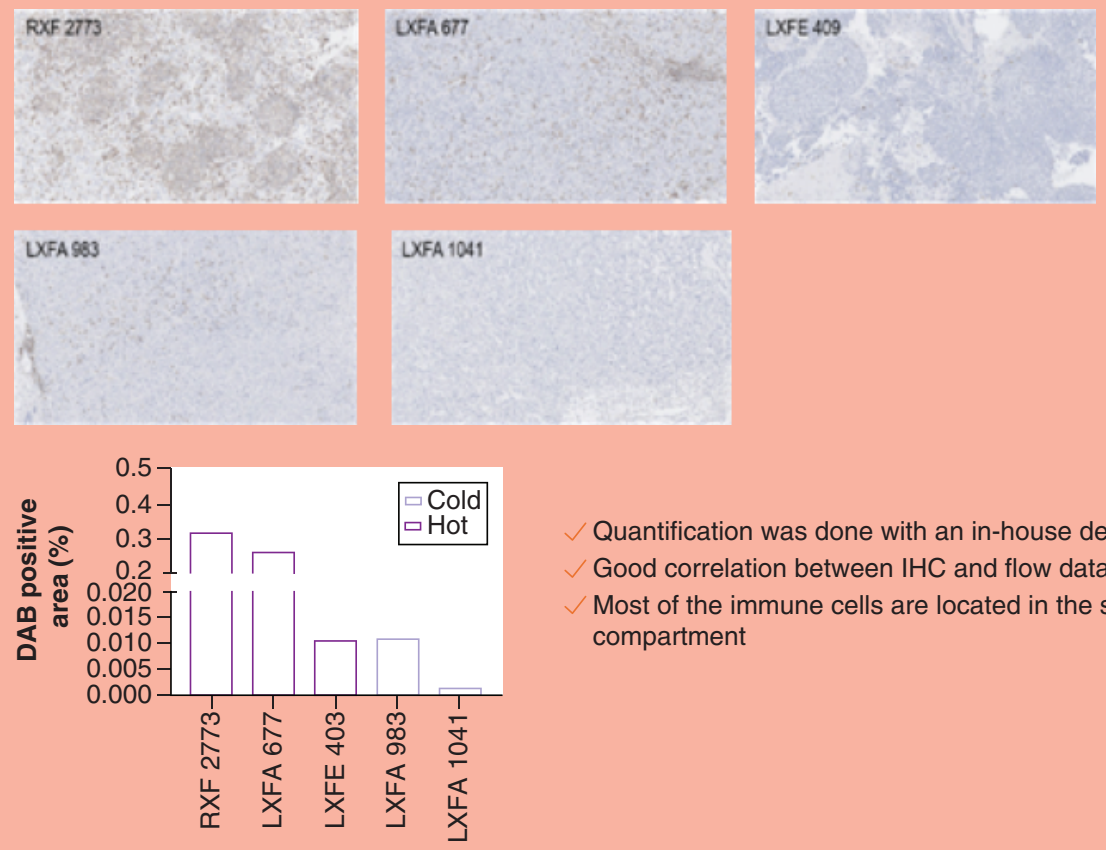

Quantification was done with an in-house developed software Good correlation between IHC and flow data

Most of the immune cells are located in the stromal compartment

Figure 5. Phenotypical and pharmacological characterization of non-small-cell lung cancer patient-derived xenograft models in humanized mice.

$* \mathrm{p}<0.05 ; * * * \mathrm{p}<0.0005$

DAB: Diaminobenzidine; FC: Fold change; IHC: Immunohistochemistry; NSCLC: Non-small-cell lung cancer; PDX: Patient-derived xenograft; TIL: Tumor infiltrating lymphocyte. 


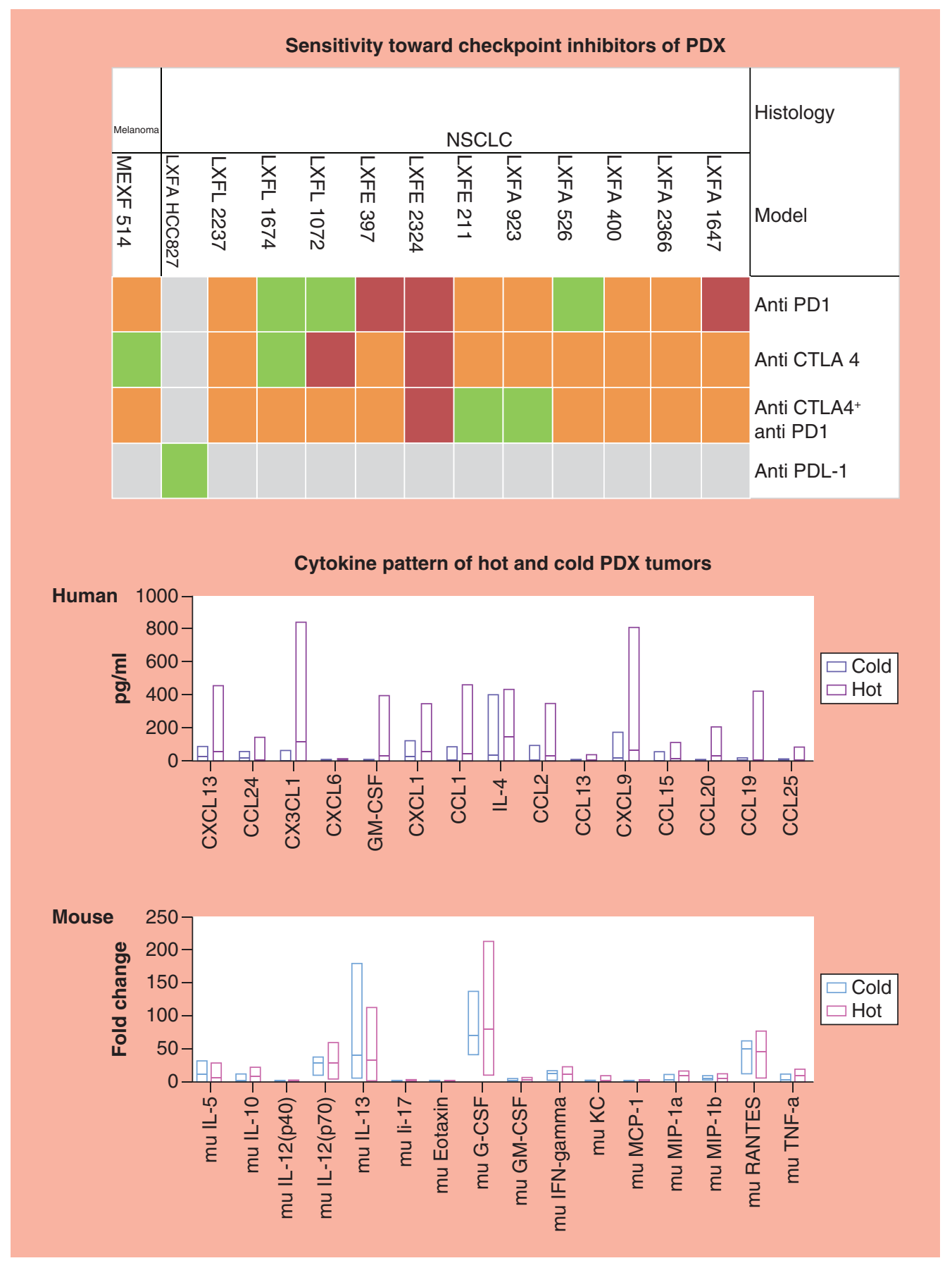

Figure 5. Phenotypical and pharmacological characterization of non-small-cell lung cancer patient-derived xenograft models in humanized mice (cont.).

$* p<0.05 ; * * * p<0.0005$.

DAB: Diaminobenzidine; FC: Fold change; IHC: Immunohistochemistry; NSCLC: Non-small-cell lung cancer; PDX: Patient-derived xenograft; TIL: Tumor infiltrating lymphocyte.

\section{Future perspective}

Compared with the rapid advance of antibody therapies, studies on immunotherapeutic small molecules have lagged far behind. However, as a modality, small molecules have ideal, proven features for cancer therapy; not only can they target immunosuppressive mechanisms similar to mAbs, but they can also stimulate intracellular 
pathways in innate or adaptive immune cells that mAbs are unable to access. This positions them uniquely as a compound class for the next generation of IO treatments which will be an exciting area in the future. A number of small molecule targets have been explored in the clinic including PD-1/PD-L1, IDO1 and STING and many other immune-therapeutic targets, such as LAG3, KIRs, TIM-3, VISTA, B7-H3 and TIGIT are currently being validated as reviewed by Chen et al. [20].

Finally, together with the emerging breadth and depth of various immunotherapeutic approaches, the number of validated and predictive in vitro and in vivo platforms will rise. Input from different disciplines beyond pharmacology and biology will be incremental to achieve this goal and contributions from physics, image analysis, bioinformatics and engineering will be essential to speed up platform development to develop innovative compounds for the benefit of the patients.

\section{Financial \& competing interests disclosure}

All authors are employees of Charles River Laboratories. The authors have no other relevant affiliations or financial involvement with any organization or entity with a financial interest in or financial conflict with the subject matter or materials discussed in the manuscript apart from those disclosed.

No writing assistance was utilized in the production of this manuscript.

\section{Open access}

This work is licensed under the Attribution-NonCommercial-NoDerivatives 4.0 Unported License. To view a copy of this license, visit http://creativecommons.org/licenses/by-nc-nd/4.0/

\section{References}

1. McIntyre P. Accelerating progress in immunotherapy. Cancer World, Milan, Italy (2016). https://cancerworld.net/cutting-edge/accelerating-progress-in-immunotherapy/

2. Kantoff PW, Higano CS, Shore ND et al. Sipuleucel-T immunotherapy for castration-resistant prostate cancer. N. Engl. J. Med. 363(5), 411-422 (2010).

3. Hollingsworth RE, Jansen K. Turning the corner on therapeutic cancer vaccines. NPJ Vaccines 4, 7 (2019).

4. Lim WA, June CH. The principles of engineering immune cells to treat cancer. Cell 168(4), 724-740 (2017).

5. Sun S, Hao H, Yang G, Zhang Y, Fu Y. Immunotherapy with CAR-modified T cells: toxicities and overcoming strategies. J. Immunol. Res. 2018, 2386187 (2018).

6. Horton BL, Fessenden TB, Spranger S. Tissue site and the cancer immunity cycle. Trends Cancer 5(10), 593-603 (2019).

7. Heubeck B, Wendler O, Bumm K et al. Tumor-associated antigenic pattern in squamous cell carcinomas of the head and neck-analysed by SEREX. Eur. J. Cancer 49(4), e1-e7 (2013).

8. Charles River Laboratories. In vitro immuno-oncology assays (2019). www.criver.com/products-services/discovery-services/in-vitro-assays/immuno-oncology?region=3696

9. Charles River Laboratories. Fully developable antibody hits? (2019). www.criver.com/insights/fully-developable-antibody-library-hits?region=3696

10. Roman S, O’Rourke M. Translational platform for immuno oncology discovery. Genetic Engineering \& Biotechnology News, NY, USA (2018). www.genengnews.com/magazine/translational-platform-for-immuno-oncology-discovery-2/

11. Liu K, Yang K, Wu B et al. Tumor-infiltrating immune cells are associated with prognosis of gastric cancer. Medicine 94(39), e1631 (2015).

12. Schmidt M, Bohm D, von Torne $\mathrm{C}$ et al. The humoral immune system has a key prognostic impact in node-negative breast cancer. Cancer Res. 68(13), 5405-5413 (2008).

13. Rongvaux A, Takizawa H, Strowig T et al. Human hemato-lymphoid system mice: current use and future potential for medicine. Annu. Rev. Immunol. 31, 635-674 (2013).

14. Zeitouni B, Tschuch C, Davis JM et al. Abstract 1840: whole-exome somatic mutation analysis of mouse cancer models and implications for preclinical immunomodulatory drug development. Cancer Res. 77(Suppl. 13), 1840 (2017).

15. Avrutskaya A, Tschuch C, Durham W et al. Abstract 3101: influence of targeted knockout of the BRCA1 gene on the pharmacologic profile of the mouse breast cancer cell line EMT6 in vitro and in vivo. Cancer Res. 78(Suppl. 13), 3101 (2019).

16. Wege AK, Schmidt M, Ueberham E et al. Co-transplantation of human hematopoietic stem cells and human breast cancer cells in NSG mice: a novel approach to generate tumor cell specific human antibodies. $m A$ bs 6(4), 968-977 (2014).

17. Sanmamed MF, Rodriguez I, Schalper KA et al. Nivolumab and urelumab enhance antitumor activity of human T lymphocytes engrafted in Rag2-/-IL2R $\gamma$ null immunodeficient mice. Cancer Res. 75(17), 3466-3478 (2015). 
18. Oswald E, Grote A, Bug D et al. Development and validation of a patient derived xenograft based preclinical platform for immuno-oncology drug development. Eur. J. Cancer 69(1), S108 (2016).

19. Bug D, Oswald E, Grote A et al. Abstract 4815: humanized single mouse trial: a preclinical platform feasible for immune-oncology drug screening and translational biomarker development. Cancer Res. 77(Suppl. 13), 4815 (2017).

20. Chen S, Song Z, Zhang A. Small-molecule immuno-oncology therapy: advances, challenges and new directions. Curr. Top. Med. Chem. 19(3), 180-185 (2019). 